\title{
Study of Deflection Routing from an Information-theoretic Perspective
}

\author{
Xinwei Zheng, Yuping Hu, Dongjun Luo and Xiaoyun $\mathrm{Wu}$ \\ School of Information Science, Guangdong University of Finance \& Economics, \\ Guangzhou, 510320, China \\ caiweihust@163.com,okhyp@gdufe.edu.cn,dongjun.luo@gdufe.edu.cn and \\ agl1975@163.com
}

\begin{abstract}
Deflection routing is an effective way to improve the efficiency and reliability of data transmission in the network. Information theory, which is the foundation of channel communications, has not yet had a direct impact on network communications. In this paper, we study the deflection routing processes of point-to-point networks from an information-theoretic perspective. It is found that the packet routing processes, which can further be divided into path determination and path switching, are quite analogous to channel communications. And such a corresponding relationship makes it possible to analyze routing processes and its performance with models and concepts in the information theory.
\end{abstract}

Keywords: Deflection routing, point-to-point networks, path determination, path switching.

\section{Introduction}

Packet contentions are inevitable in packet switching. One feasible solution without having to buffer the losing packet is to use deflection routing. In contrast to "store-andforward" routing where the network allows buffering, deflection routing, also known as hot potato routing [1], is a routing scheme without buffering and can only be implemented for packet switched network. In case the individual communication links cannot support more than one packet at a time, excessive packets will be transferred to other available links.

Deflection routing utilizes idle links of a network as temporary storages, which essentially exploit the inherent path diversity of the network. However, most researches prefer using experiments or simulation to investigate deflection routing. There is a lack of theoretical analysis on the model of the relationship between path diversity and network topology and routing policies. As the data transmission in networks has a close relationship with the information theory, some researchers extend the model and methods of the information theory from channel communication to networks. E. Ayanoglu [2] used the diversity coding which is similar to that of the linear coding theory. S. Pradhan [3] took a method similar to maximum-distance separable erasure channel codes.

Inspired by these efforts, we try to investigate the deflection routing from an information transmission perspective and find the corresponding relationship between deflection routing processes and channel communications. Due to the complexity of network topology, we start with the research from the simplest case, namely point-topoint networks. 
The rest of the paper is organized as follows. In Section 2, we study deflection routing processes for point-to-point networks from an information-theoretic perspective. Some related work is introduced in Section 3. And a brief summary is given in Section 4.

\section{Point-to-point Networks}

To simplify the analysis, the routing process is further being decomposed logically into two sub-processes, path determination (PD) and path switching (PS).

Path determination (PD) is a process executed at nodes with routing ability. The output of PD for each packet is a specific path to the destination or to the next hop, which is named the default path (or expected path). The primary function of PD is to allocate a feasible default path for a routing request. As there are more than one routing requests from different data sources simultaneously, PD has to insure to avoid internal contention for paths among these requests.

Path switching (PS) is a process to transit a data packet hop-by-hop to the destination. In networks without path interferences, for each routed packet, the actual path is exactly the default path allocated in PD processes. In networks with interferences, the output of PD may be unavailable due to unexpected bursty interferences. Network nodes with routing capability, such as routers, etc., may deflect the packet to alternative paths if available, or just drop it. Therefore, interferences occurred stochastically make the actually transportation paths deviate the default paths. The deviation is completely due to interferences and corresponding countermeasures taken by routing nodes, such deflection routing. The objective of PS is to transport packets to their destination even under unexpected interferences.

The path interferences discussed here specifically refer to external disturbances against normal routing operations, which include unexpected links' failures, traffic congestions, physical circuit-cuts, fiber-cuts, wireless fading, etc. The causes and impacts of routing interferences and ordinary channel noises are quite different. The impacts of noises focus on physical layer, and cannot be mitigated or eliminated by changing routing policies. However, the impacts of path interferences focus on network layer, and can be effectively avoided or lessened by countermeasures taken at the network layer.

\subsection{Path Determination}

For a packet to be sent from source node, there exist two types of uncertainty, namely temporal uncertainty and spatial uncertainty. The former one represents the time slot taken by a packet, which is determined at the transport layer. The latter one represents the path should be taken, which is determined by routing policy at the network layer.

In random multi-path routing, the output of path determination can be depicted as a random variable. During routing processes, when facing two available paths as A and B, the routing decision for a data packet is either A or B. For the destination side, the reception of a data packet, together with the path information it takes, eliminate a type of uncertainty, which is called path uncertainty.

Therefore, according to the definition of information provided by Shannon's information theory, path determination is actually an information source $\mathrm{X}$ which generates information on path allocation. The source which generates path information is routing information source (RIS).

For a single-hop network with $\mathrm{r}$ candidate paths at the source node, we denote the probability distribution of path usage as

$$
P=\left\{p_{1}, p_{2}, \ldots, p_{r}\right\}, \sum_{i=1}^{r} p_{i}=1 .
$$

The information generated by each path determination is 


$$
I\left(a_{i}\right)=-\log _{2} p_{i}(i=1, \ldots N) .
$$

$I\left(a_{i}\right)$ Is the amount of self-information generated by one specific path, to describe the overall information generated by path determination $X$, it is necessary to make an average of $I\left(a_{i}\right)$ in the probability space:

$$
H(X)=-\sum_{i=1}^{r} p_{i} \log p_{i}
$$

$H(X)$ Is the average information entropy of path determination $X$.

For a network without interference, the output from the source node represents the actual transmission path. For instance, if the path $a_{i}$ has been chosen as the default path with probability $p_{i}$, the source node will send the packet to path $a_{i}$ with probability $p_{i}$. This will lead to the result that the destination receives packet from the path $a_{i}$ with probability $p_{i}$. Therefore, in disturbance-free network, the probability distribution at destination will be exactly the same as the probability distribution at the source node. The network routing problem can be simplified as the path determination problem at the source node. The principal purpose of path determination is to avoid contention for resources among internal routing requests.

However, in actual circumstances, quite much interference such as network link failures, traffic congestions, malicious external attacks, the sudden disruption, wireless fading, etc., cannot be avoided completely. In the face of unexpected interference, if the default path is unavailable, network nodes are usually used deflection routing to bypass the failed components. With consideration of the randomness of path interferences, deflection routing produces additional uncertainty to the actual transmission path, which affects the path switching process.

\subsection{Path Switching}

To achieve a successful packet routing, agreement on which path should be used by the routing request has to be reached beforehand through negotiations between source and destination using routing protocols. Only when the path the destination receives from matches the very path the source sends to, can the packet be successfully routed.

All viable outgoing paths at the source node constitute the input set of path switching. All possible receiving paths constitute the output set. If there are no interferences, path switching process is essentially a path bijection between the source and destination. Therefore, routing issues at the network layer can be simplified as path determinations at the source end.

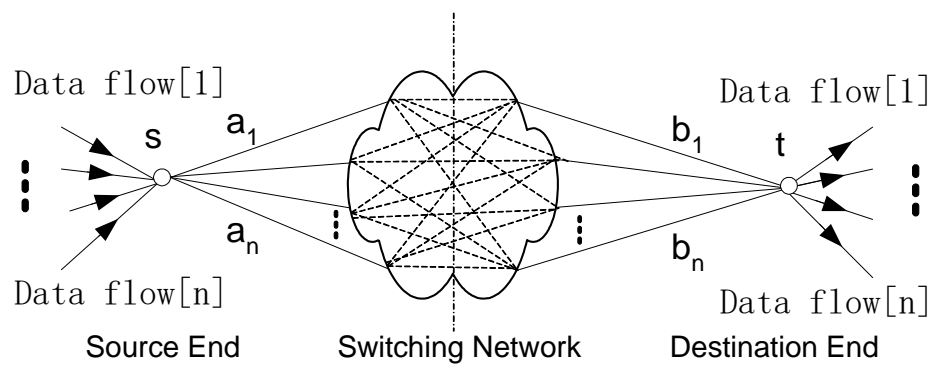

Figure 1. Path Switching in Point-to-point network 
However, in practice, unpredictable path interferences cannot be avoided completely. The actual paths that data packets used are not always the default paths allocated by path determination. With deflection routing strategy, once a default path is not available due to interferences, data packet should be transmitted on alternative paths, or to just be dropped. Therefore, stochastic path interferences usually make the path switching no longer a path bijection mapping, but a path mapping based on a probability distribution.

To maximize the network performance, the output of path determination should match the input of path switching. This involves two levels of matching relations. First, each viable output of path determination should also be a viable input of path switching. Secondly, the probability distribution of path determination output should match the network property of path switching process, and makes the best switching performance.

\subsection{Transition Probability Function of Path Switching}

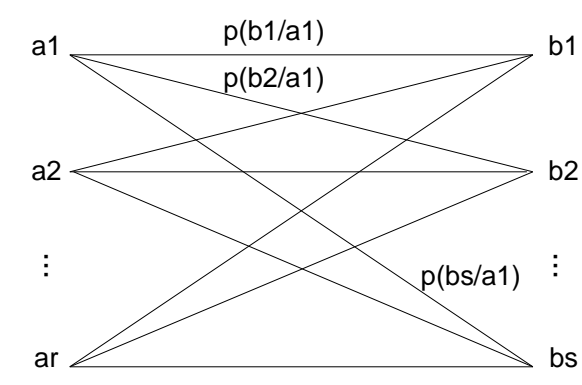

\section{Figure 2. Transition Probabilities for Inputs and Outputs of Path Switching}

This transition probability function depicts the effects of the interferences on the network.

In a single hop network, denote the output set of path determination as $X:\left\{a_{1}, a_{2}, \ldots a_{r}\right\}$, which is also the input set of path switching. Denote the receiving path set of destination end as $Y:\left\{b_{1}, b_{2}, \ldots b_{s}\right\}$, which is also the output set of path switching. The mathematical model of path switching can be represented as:

$$
P(Y \mid X):\left\{p\left(b_{j} \mid a_{i}\right)(i=1, \ldots, r ; j=1, \ldots s)\right\},
$$

where $p\left(b_{j} \mid a_{i}\right)$ is the transition probability function of path switching process. If $(r \times s)$ transition probability functions queue up, we get the transition probability matrix as follows.

$$
[P]=\left[\begin{array}{cccc}
p\left(b_{1} \mid a_{1}\right) & p\left(b_{2} \mid a_{1}\right) & \ldots & p\left(b_{s} \mid a_{1}\right) \\
p\left(b_{1} \mid a_{2}\right) & p\left(b_{2} \mid a_{2}\right) & \ldots & p\left(b_{s} \mid a_{2}\right) \\
\ldots & \ldots & \ldots & \ldots \\
p\left(b_{1} \mid a_{r}\right) & p\left(b_{2} \mid a_{r}\right) & \ldots & p\left(b_{s} \mid a_{r}\right)
\end{array}\right]
$$

It is evident that transition probability matrix $[P]$ of path switching is exactly the same as that of channel communication. 


\subsection{Mutual Information of Path Switching}

As path determination and path switching can be modeled as the information source and the information channel respectively, we define the mutual information for path switching, which is quite similar to that of channel communication.

Given the probability space of path determination as follows:

$$
[X \cdot P]:\left\{\begin{array}{ccccc}
X: & a_{1} & a_{2} & \ldots & a_{r} \\
P(X): & p\left(a_{1}\right) & p\left(a_{2}\right) & \ldots & p\left(a_{r}\right)
\end{array}\right\} .
$$

Assume the set of path determination output complies with the available path set of the network, each output of path determination $a_{i}(i=1,2, \ldots, r)$ can be input into the path switching matrix, and then correspond to an actual path $b_{j}(j=1,2, \ldots, s)$. Assume the prior uncertainty is

$$
I\left(a_{i}\right)=-\log p\left(a_{i}\right) \quad(i=1,2, \ldots, r) .
$$

Having received packet from the path $b_{j}$, the probability that the packet was sent from path $a_{i}$ is $p\left(a_{i} \mid b_{j}\right)$. The posterior uncertainty is:

$$
I\left(a_{i} \mid b_{j}\right)=-\log p\left(a_{i} \mid b_{j}\right), \quad(i=1,2, \ldots r ; j=1,2, \ldots, s) .
$$

Therefore, the uncertainty eliminated by path switching is:

$$
\begin{aligned}
I\left(a_{i} ; b_{j}\right) & =I\left(a_{i}\right)-I\left(a_{i} \mid b_{j}\right) \\
& =-\log p\left(a_{i}\right)-\left[-\log p\left(a_{i} \mid b_{j}\right)\right] \\
& =\log \frac{p\left(a_{i} \mid b_{j}\right)}{p\left(a_{i}\right)} \quad(i=1,2, \ldots, r ; j=1,2, \ldots, s) .
\end{aligned}
$$

Above equation represents the amount of path information actually received by destination side in the path switching process. We define it as the mutual information of path switching.

Similar to mutual information defined for channel communication, the mutual information of path switching represents the reduction in path uncertainty due to path switching. $I\left(a_{i} ; b_{j}\right)$ is the mutual information function.

From conditional probability formula, we have:

$$
\begin{aligned}
I\left(a_{i} ; b_{j}\right) & =\log \frac{p\left(a_{i} \mid b_{j}\right)}{p\left(a_{i}\right)}=\log \frac{p\left(a_{i} b_{j}\right)}{p\left(a_{i}\right) p\left(b_{j}\right)} \\
& =\log \frac{p\left(b_{j} \mid a_{i}\right)}{p\left(a_{i}\right)} \\
& =I\left(b_{j} ; a_{i}\right)
\end{aligned}
$$


The above equation shows that information about $a_{i}$ obtained from $b_{j}$, equals to information about $b_{j}$ obtained from $a_{i}$.

The average mutual information in joint probability space is:

$$
I(X ; Y)=\sum_{i=1}^{r} \sum_{j=1}^{s} p\left(a_{i} b_{j}\right) I\left(a_{i} ; b_{j}\right)
$$

According to the symmetry property of mutual information, we have

$$
I(X ; Y)=I(Y ; X) .
$$

From the definition of average mutual information, it is easy to derive conclusions as follows.

Corollary 1: $I(X ; Y)=H(X)-H(X \mid Y)$ where $H(X)$ is the entropy of path determination, $H(X \mid Y)$ is the conditional entropy which represents the average uncertainly of $X$ with the knowledge of $Y \cdot H(X \mid Y)$ is also called average equivocation.

Corollary 2: $I(X ; Y)=H(X)+H(Y)-H(X Y)$, where $H(X Y)$ is the joint entropy of $X$ and $Y$.

Corollary 3: $I(X ; Y)=H(Y)-H(Y \mid X)$, where $H(Y \mid X)$ represents the average uncertainty of $Y$ with the knowledge of $X . H(Y \mid X)$ is actually caused by network interference, and is called average entropy of interference.

The average mutual information has following properties.

Property 1 (Nonnegativeness): $I(X ; Y) \geq 0$, when $\mathrm{X}$ and $\mathrm{Y}$ are independent of each other, the equation holds.

Property 2 (Extremum property):

$$
I(X ; Y)=H(X)-H(X \mid Y) \leq H(X) .
$$

When $H(X \mid Y)=0, I(X ; Y)=H(X)$.

\subsection{Path Switching Capacity}

Definition (Path Switching Rate): Average mutual information of path switching $I(X ; Y)$ represents the average amount of information transmitted by each successful packet routing, which is also called path switching rate, noted as $R(X, Y)$.

Definition (Path Switching Capacity): Path switching capacity is the maximum rate at which path information can be sent over the network and rebuild the information at the output with a vanishingly low probability of error.

$$
C=\max _{P(X)}\{I(X ; Y)\} .
$$


Generally speaking, the average mutual information is the function of the probability distribution of path determination

$$
p\left(a_{i}\right)(i=1,2, \ldots, r),
$$

and the transition probability function

$$
p_{0}\left(b_{j} \mid a_{i}\right)(i=1,2, \ldots, r ; j=1,2, \ldots, s),
$$

which is

$$
I\left[p\left(a_{i}\right), p\left(b_{j} \mid a_{i}\right)\right] .
$$

Define (Matched Path Determination): A path determination which can maximize the path switch capacity $C$, is called the matched path determination.

For the matched path determination, some related conclusions are as follows.

Proposition (Matched Path Determination Policy): Given a single-hop point-topoint network, its routing mutual information reaches the path switching capacity if and only if the probability distribution of routing policy $P(X):\left\{p_{1}, p_{2}, \ldots, p_{r}\right\}$ satisfied the follow conditions.

$$
\begin{aligned}
& I\left(a_{i} ; Y\right)=C, \text { for i with } p_{i} \neq 0, \\
& I\left(a_{i} ; Y\right) \leq C, \text { for i with } p_{i}=0 .
\end{aligned}
$$

\section{Related Work}

In [4], A. Ephremides stressed that information theory has not yet made a comparable mark in the field of communication networks. There are increasing evidences that Shannon's work on point-to-point communication can be brought about on the field of network communication.

Inspired by A. Emphremides' idea, T.T. Lee [5] gave a detailed comparison between packet switching and information channel communication. Analogies between packet switching and information transmission were listed. However, the comparison was performed based on packet-switched CLOS network, and most analysis was limited to continuous cases. No discrete cases were mentioned. In this paper, we focus on network layer of point-to-point network, start with discrete cases and exploit the routing mechanism from information-theoretic perspective.

In [6], path switching was defined as a quasi-static routing scheme, which was actually a compromise of static routing and dynamic routing. In [7], path switching is defined as a mechanism that allows a source end system to dynamically switch among multiple paths to a destination. In [8], revised path switching scheme is proposed, which takes the application-layer factors into account, and dynamically decides the best performing path for each voice call. Obviously, these path switching definitions are quite different from ours. In this paper, we articulate the essential similarities between path switching in network routing and data transmission in channel communication.

\section{Summary}

In this paper, we focus on the deflection routing mechanism of point-to-point networks, and study the essential analogies between network routing and channel communication, 
which make it possible to analyze routing processes with rich models and concepts from information theory.

However, the discussion in this paper is only limited to the simplest cases of point-topoint networks with completely disjoint paths. The future job is to extend the discussion to networks with complex topologies and partially disjoint paths.

\section{Acknowledgements}

This work was supported by Humanities and Social Sciences Planning Fund of Ministr y of Education (No.13YJAZH099), Guangdong University of Finance \& Economics Foun dation (No.11ZD52001).

\section{References}

[1] P. Baran, "On Distributed Communications Networks", Communications, IEEE Transactions on [legacy, pre-1988], vol. 12, (1964), pp. 1-9.

[2] E. Ayanoglu, "Diversity coding for transparent self-healing and fault-tolerant communication networks", IEEE Transactions on Communications, vol. 41, (1993), pp. 1677-1686.

[3] S. S. Pradhan, R. Puri, and K. Ramchandran, "n-channel symmetric multiple descriptions-part I:(n, k) source-channel erasure codes", Information Theory, IEEE Transactions on, vol. 50, (2004), pp. 47-61.

[4] A. Ephremides and B. Hajek, "Information theory and communication networks: an unconsummatedunion", Information Theory, IEEE Transactions on, vol. 44, (1998), pp. 24162434.

[5] T. T. Lee, "The Mathematical Parallels Between Packet Switching and Information Transmission", Arxiv preprint cs.IT/0610050, (2006).

[6] T. T. Lee and C. H. Lam, "Path switching-a quasi-static routing scheme for large-scale ATMpacket switches", Selected Areas in Communications, IEEE Journal on, vol. 15, (1997), pp. 914-924.

[7] S. Tao, K. Xu, Y. Xu, T. Fei, L. Gao, R. Guerin, J. Kurose, D. Towsley, and Z. L. Zhang, "Exploring the performance benefits of end-to-end path switching", Proceedings of IEEE ICNP, (2004).

[8] S. Tao, K. Xu, A. Estepa, T. Fei, L. X. Gao, R. Guerin, J. Kurose, D. Towsley, and Z. L. Zhang, "Improving VoIP quality through path switching", in Ieee Infocom 2005: the Conference on Computer Communications, Vols 1-4, Proceedings, K. Makki and E. Knightly, Eds., ed Los Alamitos: Ieee Computer Soc, (2005), pp. 2268-2278.

\section{Authors}

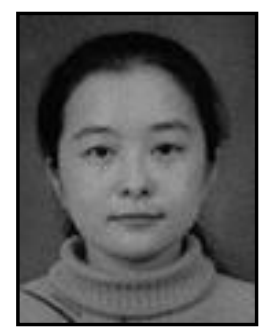

Xinwei Zheng is an instructor in Information Science School at Guangdong University of Business Studies. She received her Master and PHD degree in Computer Science from Huazhong University of Science and Technology in 2003 and 2008. Her current research interests include Multipath Routing, computer network technology and stream media technology.

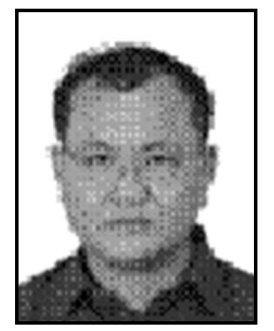

Yu-Ping Hu received his B.S. degree in celestial survey from Chinese Academy of Science, China, in 1996 and his Ph.D. degree in computer science from Huazhong University of Science and Technology, Wuhan, China, in 2005.He is a professor in Guangdong University of Finance \& Economics since 2006. His current research interests include digital watermarking, image processing, multimedia and network security. 


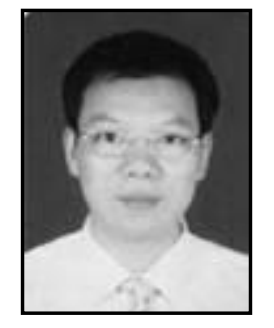

Donjun Luo received his M.S. degree in Computer Software and Theory from Sun Yat-Sen University of Guangzhou, in 2004 and $\mathrm{Ph}$.D. degree in Information Security from South China University of Technology of Guangzhou in 2014. His main research interests include trusted computing and cloud computing security.

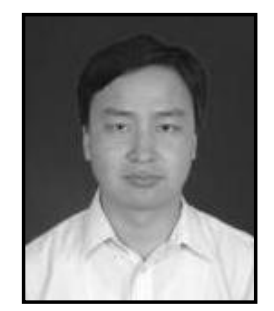

Xiaoyun Wu received the Ph.D. degrees from Sun Yat-Sen University, Guangzhou, China, in 2006. He is currently an Associate Professor with School of Information Science, Guangdong University of Finance \& Economics. His current research interests include image processing and information security. 
International Journal of Future Generation Communication and Networking Vol. 8, No. 1 (2015) 